\title{
Parasitic co-infections: challenges and solutions
}

\author{
EDITED By MARK BOOTH, ANDREA GRAHAM AND MARK VINEY \\ CO-ORDINATING EDITOR L. H. CHAPPELL
}

\section{Preface}

Co-infections are the norm in nature, yet rare in the laboratory. Fortunately, the latter pattern is beginning to change, and laboratory studies comparing co- and mono-infections have shown that many aspects of both parasite and host biology differ between these two settings. But, whether and how the interactions revealed in the laboratory affect natural populations is still unclear. The papers in this volume seek to capture the current state of this field.

Jerzy Behnke and Sandra Telfer have looked for, and found, evidence of the effects of co-infection on parasites and on animal hosts in the wild. Jan Bradley's work has looked at the immuno-biology of geohelminth co-infection in humans. In all cases, there is evidence for effects of co-infection but the extent to which these effects structure the observed parasite fauna remains unknown. All three authors propose further studies to elucidate how interactions that have been described in the laboratory might operate in nature.

One potential obstacle to detecting effects of co-infection in nature is that parasites may interact in complex ways that defy intuition. Co-infection biology is therefore ripe for theoretical or mathematical approaches, several of which are included in this volume. One challenge, considered by Jo Lello, is to find general rules that apply to broad parasite groups (defined taxonomically or by 'functional' criteria) and are predictive of the clinical outcome of co-infection. A further challenge is to predict the epidemiological and evolutionary consequences of co-infection. In this vein, Andy Fenton considers the population-level impact of mutual inhibition between $\mathrm{T}$ helper type 1 (Th1) and Th2 immune responses, while Jamie Lloyd-Smith addresses how HIV may affect the emergence of new parasite strains. Each of these theoretical contributions forges a strong link with empirical data for future investigation.
Co-infections are of applied relevance to human health: co-infections may have an additive or multiplicative effect in enhancing or ameliorating disease. Co-infections may also affect the outcomes of control programmes. Several authors consider these issues, focusing on common and debilitating infections of human hosts: three helminth species account for the loss of 39 million disability adjusted life years (DALYs), and malaria for the loss of 36 million DALYs. With this perspective, Simon Brooker considers how malaria-helminth co-infection may affect disease severity. Mathieu Nacher describes his investigations of the effect of geohelminth infections on both mild and severe malaria in adults. Franca Hartgers presents field immunology data showing how helminth infections may modulate immune responses towards malaria in school-aged children. Whether or not the underlying mechanisms are as described in the laboratory, the patterns observed in these field studies merit close attention.

This wide-ranging collection of papers addresses core questions of co-infection biology from different and complementary perspectives. Ideally, all areas will progress in an integrated manner. For example, both the theoretical work and the field work described in this volume raise further questions for laboratory scientists to consider. Despite increasing activity in recent years, co-infection biology is very much in its infancy. These studies and approaches will, we hope, help to set the future research agenda.

We would like to thank Cambridge University Press for their continued and generous financial support of this symposium.

MARK BOOTH

ANDREA GRAHAM

MARK VINEY

March 2008 